\title{
Advances in Serial-Section Broad-Ion-Beam Tomography
}

\author{
T. Hosman ${ }^{1}$, S. Coyle ${ }^{1}$, M. Hassel-Shearer ${ }^{1}$ and J.A. Hunt ${ }^{1}$; A. Gholinia ${ }^{2}$, P. Withers ${ }^{2}$ \\ ${ }^{1}$ Gatan Inc., 5794 W. Las Positas Blvd., Pleasanton, CA 94588 USA \\ ${ }^{2}$ School of Materials, University of Manchester, Manchester, M1 7HS, UK
}

The Gatan IPrep milling system ${ }^{1}$ enables tomographic reconstruction of a wide range of materials and biological samples using broad-ion-beam serial-sectioning (SSBIB) - alternately removing material by ion-polishing and imaging the remaining polished surface with optical or scanning electron microscopy (SEM). ${ }^{2}$ Under computer control it is practical to acquire 2-D images of hundreds of sections without user intervention and assemble them for 3-D visualization and measurements. Broad-ion-beam milling has the ability to efficiently acquire tomographic data of several $\mathrm{mm}^{2}$ areas because of its intense and largediameter beam size and maintain excellent image quality by utilizing low-energy ions. In general, the resulting 3-D images permit significantly better understanding of the structure of complex systems than would be possible with a few strategically acquired 2-D images acquired at various depths.

In the current IPrep implementation, the BIB system is mounted to the side of an SEM chamber and isolated by a gate valve to prevent contamination of the SEM system from milling effluent. The sample is exchanged between the chambers by a linear transfer stage. A dock on the SEM stage receives the sample and clamps it kinematically for highly-repeatable alignment between successive sample exchanges.

Recent advances in SSBIB development have improved understanding and control over the repeatability and uniformity of increments in polishing depth, along with the option for sample coating after each slice is milled. Software advances now routinely permit multi-scale and multi-technique imaging. Developments in progress are expected to permit analysis areas of tens of $\mathrm{mm}^{2}$ per slice.

SSBIB was used to analyze a plated Aluminum 6061-T6 machined part used in a cryogenic application with the goal of understanding the cause of poor surface qualities pre- and post-plating, and the role and nature of agglomerations of precipitates seen with 2-D light microscopy and SEM. Aluminum 6000 series alloys have excellent heat transfer and mechanical strength properties permitting use in extreme cryogenic environments while maintaining dimensional integrity during repeated thermal cycling. They are strengthened by precipitation hardening where $\mathrm{Mg}_{2} \mathrm{Si}$ precipitates play a crucial role. ${ }^{3}$

In this work, problems with surface plating were shown to originate from larger iron-rich precipitates (IRP) that align in chain-like structures requiring a field-of-view (FOV) of several hundred microns to visualize. Fig. 1 shows a sub-volume of $434 \times 356 \times 441 \mu \mathrm{m}^{3}$ acquired in $500 \mathrm{~nm}$ slices. Milling time was 5 minutes/slice using $8 \mathrm{keV}$ Ar-ions incident at $5^{\circ}$, which produces milling rates of $>5 \mathrm{e} 6 \mathrm{um}^{3} /$ hour and $>$ $1 \mathrm{~mm}^{2}$ quality areas.

At each slice, 2 different FOV were imaged with both SE and BSE detectors to create $4 \mathrm{~K} \times 4 \mathrm{~K}$ images in 5 minutes each (only the smaller FOV shown). With overhead, data from approximately 70 slices per day was acquired. Data acquisition does not necessarily have to be continuous, as was the case with this sample -- acquisition can be paused so the SEM can be used for other samples.

Fig. 1a shows a 3D rendered BSE contrast sub-volume. The Al matrix grains are grey, IRP are brightwhite and $\mathrm{Mg}_{2} \mathrm{Si}$ precipitates are darker-white. Top down the IRP appear randomly oriented, whereas side views hint at a vertical chain alignment. Fig. $1 \mathrm{~b}$ and higher mag Fig. 1c are precipitate-only renderings that more clearly shows vertical chain alignment. Fig. 1d is a top-down projection of all IRP (green) and $\mathrm{Mg}_{2} \mathrm{Si}$ (dark blue) precipitates obtained by thresholding the BSE signal for each phase. Areas of light blue show regions free of precipitates and indicate the substantial degree of vertical alignment. In Fig. 1e, 
X-ray compositional maps of a separately ion-milled cross-section of the same sample show IRP are shown to be much larger size and total volume than $\mathrm{Mg}_{2} \mathrm{Si}$ precipitates.

[1] T. Hosman et al., Microsc. Microanal. 22:3, (2016), p590-1.

[2] Winiarski et al., Ultramicroscopy 172, (2017) p52-64.

[3] Hatch JE, editor. Aluminum: properties and physical metallurgy. ASM International, (1984), p64.

(a)

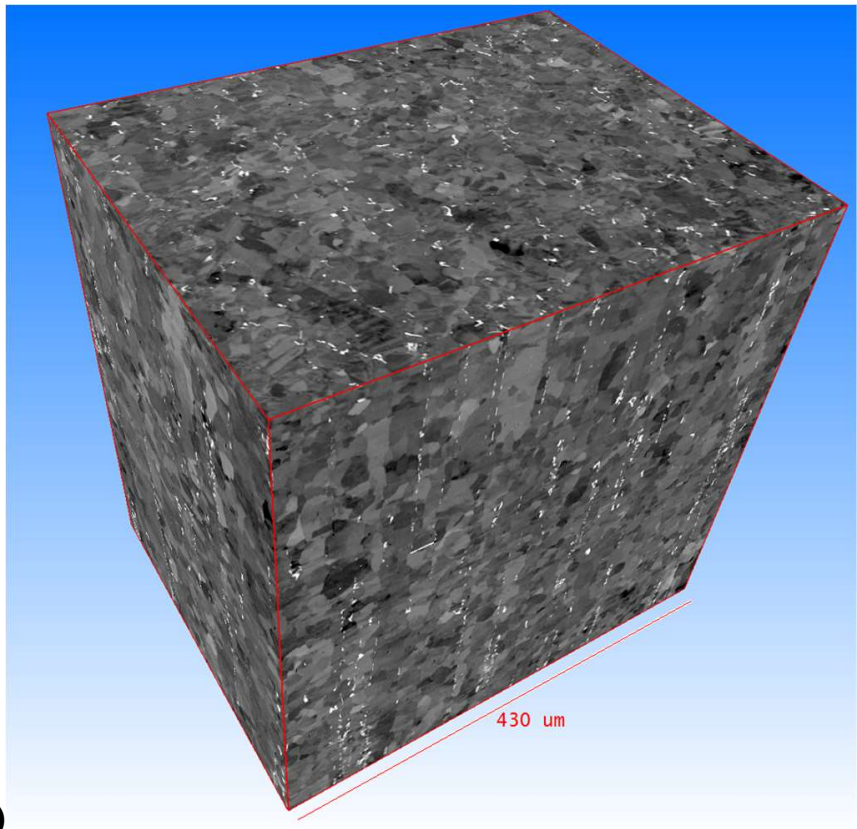

(b)

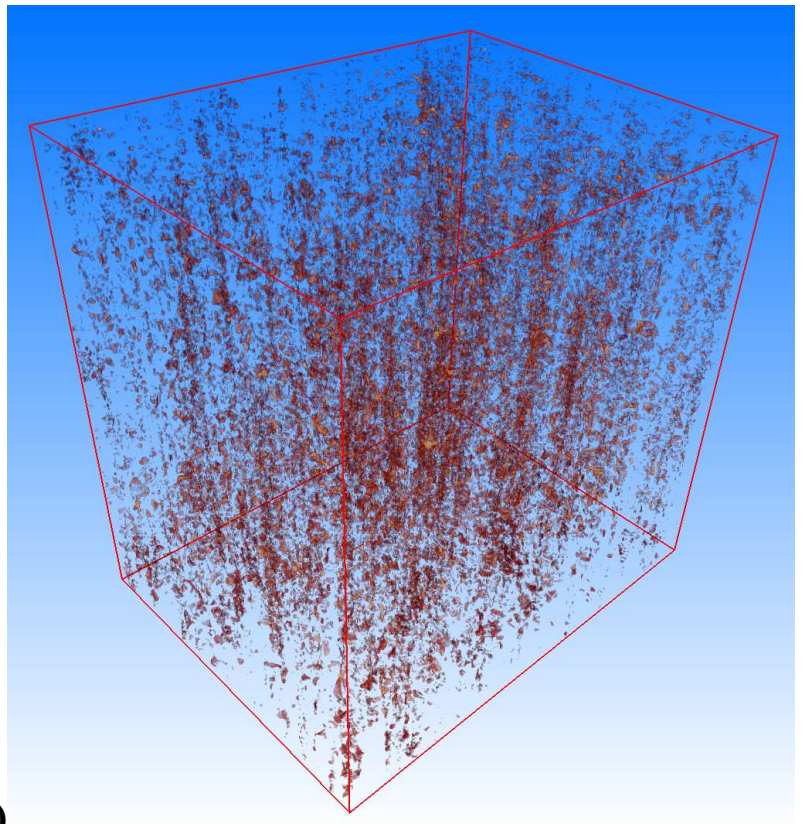

(c)

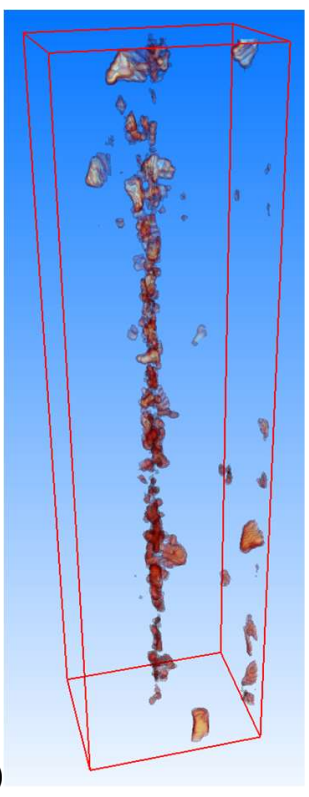

(d)

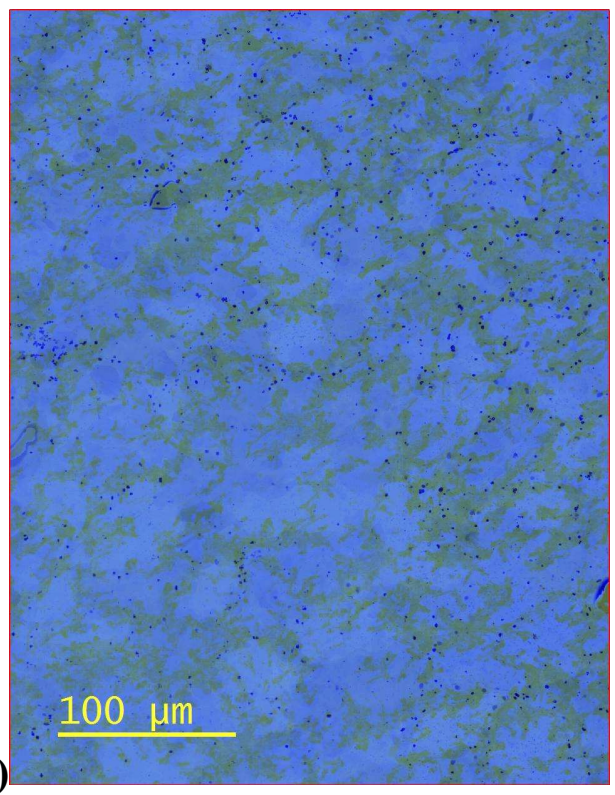

(e)

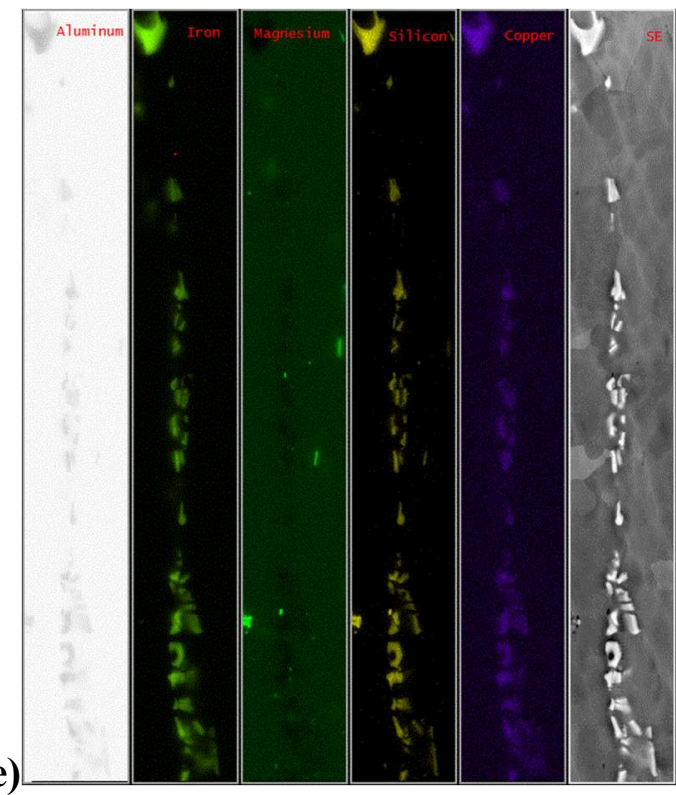

Figure 1. (a) BSE rendered volume, (b) precipitate-only rendering, (c) high-mag precipitate-only rendering, (d) top-down projection showing precipitate-free areas, (e) x-ray compositional maps 